\title{
28 Research Suare \\ Urine Output Calculated Using Actual Body Weight may Result in Overestimation of Acute Kidney Injury for Obese Patients
}

\section{Jun Jiang}

Zhongnan Hospital of Wuhan University

Jing Zhang

Zhongnan Hospital of Wuhan University

Ye Liu

Zhongnan Hospital of Wuhan University

Dongxue Xu

Zhongnan Hospital of Wuhan University

Zhi-Yong Peng ( $\nabla$ pengzy5@hotmail.com )

Wuhan University Zhongnan Hospital https://orcid.org/0000-0002-3873-9607

Research

Keywords: AKI, body weight, mortality

Posted Date: December 11th, 2020

DOI: https://doi.org/10.21203/rs.3.rs-125411/v1

License: (9) This work is licensed under a Creative Commons Attribution 4.0 International License.

Read Full License 


\section{Abstract}

Background: The derived hourly urine output (UO) indexed by body weight is one of the major criteria for diagnosis of acute kidney injury (AKI). However, it is unknown whether actual body weight (ABW) or ideal body weight (IBW) should be used. This study aims to explore whether UO calculation based on ABW might lead to overestimation of AKI.

Methods: AKI patients identified in the MIMIC database by different components of the KDIGO guidelines and different definitions of body weight were retrospectively studied. We compared hospital and 90-day mortality to decide whether AKI patients diagnosed by ABW- or IBW-normalized UO had the same outcome.

Results: In the cohort of 14,725 patients, AKI was identified in 4,298 $(29.19 \%)$ and 3,060 (20.78\%) patients respectively when ABW or IBW was used to calculate hourly UO $(p<0.001)$. AKI stages differed between these patients $(p<0.001)$ with a kappa of $0.87(95 \% \mathrm{Cl}, 0.86 \sim 0.88)$ and an agreement percentage of $90.40 \%$. Multivariate logistic regression revealed AKI patients identified by UO calculated from ABW had similar hospital and 90-day mortality to that of patients with no evidence of AKI. Whereas AKI patients identified by SCr or by both ABW and IBW had over twice higher the risks of hospital death, 1.7 and 1.4 times higher the risks of 90-day death respectively compared with patients with no evidence of AKI. Results were confirmed in sensitivity analysis where patients whose admission creatinine levels were within the normal reference ranges and sepsis patients were studied.

Conclusions: Calculating hourly body weight normalized UO using ABW may lead to underestimation of $\mathrm{UO}$ and overestimation of AKI.

\section{Introduction}

Acute kidney injury (AKI) has been reported to occur in over $50 \%$ of the critical ill and associated with increased mortality ${ }^{1}$. Although several definitions and guidelines have been proposed over the past few decades, the Kidney Disease Improving Global Outcomes (KDIGO) criteria have been widely accepted by researchers and applied in clinical practice around the world ${ }^{2}$. KDIGO defines AKI by both alternations in serum creatinine ( $\mathrm{SCr}$ ) levels and body weight normalized hourly urine output (UO).

However, the KDIGO guideline failed to specify whether actual body weight (ABW) or ideal body weight (IBW) should be used when calculating body weight normalized $\mathrm{UO}^{3}$. Therefore, the questions of which body weight definition would more suitable and should be used for AKI diagnosis and further, whether there would be any difference between AKI patients identified by the two different body weights arose ${ }^{4-6}$. For obese and underweight patients, ABW and IBW could differ significantly, leading to differences between $U O$ calculated from them and thus might result in discrepancy regarding diagnosis and staging of AKI. Specifically, ABW is usually bigger than IBW for an obese patient. When calculating hourly UO, using $A B W$ rather than IBW tends to yield smaller values, more likely to be lower than the thresholds 
defined by KDIGO guidelines. As a result, an obese patient is more likely to be diagnosed as AKI when using ABW instead of IBW to calculate body weight normalized hourly UO.

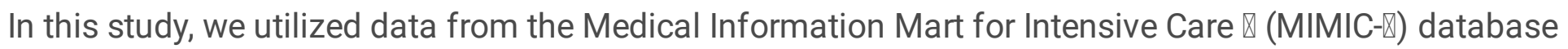
and explored differences between patients diagnosed by different components of the KDIGO guidelines and different definitions of body weights. We hypothesized that using ABW might lead to underestimation of patients' hourly UO and subsequently overestimation of AKI.

\section{Methods}

\section{Study Population}

We used data from the MIMIC- $\llbracket$ database v1.4, a collaboration between the Beth Israel Deaconess Medical Center (BIDMC) and the Laboratory for Computational Physiology at the Massachusetts Institute of Technology (MIT $)^{7}$. It is a single-center database containing 38,597 distinct patients and 49,785 hospital admissions between 2001 and 2012 at BIDMC, a 700-bed teaching hospital of Harvard Medical School in Boston, Massachusetts with 77 adult intensive care unit (ICU) beds. Data in the database includes patients' vital signs, laboratory tests, observations and notes charted by care providers, fluid balance, procedure codes, diagnostic codes, imaging reports, length of hospital stay, and survivals. All patients in the database were deidentified and analysis of the data is unrestricted once a data use agreement is accepted.

To ensure independence of between hospital admissions, we only included the first ICU stay of each patient. ICU stay records shorter than 24 hours or without adequate records of $\mathrm{UO}$ and $\mathrm{SCr}$ (less than 2 available measurements of both) were also excluded. An age of older than 89 years was shifted and thus not available in the database, so we only included patients with an age between 18 and 89 years. We then dropped all patients whose body weight or height were not documented within first day of their ICU stay. Patients diagnosed with end stage renal disease (ESRD) as documented by the International Classification of Diseases 9th Revision (ICD-9) codes were also excluded.

\section{AKI definition and grouping of patients}

AKI was diagnosed according to KDIGO guidelines: increase in serum creatinine $(\mathrm{SCr})$ by $\geq 0.3 \mathrm{mg} / \mathrm{dl}$ $(26.5 \mu \mathrm{mol} / \mathrm{I})$ within 48 hours or increase in $\mathrm{SCr} \geq 1.5$ times of baseline which is known or presumed to have occurred within the prior 7 days, or urine volume $\leq 0.5 \mathrm{ml} / \mathrm{kg} / \mathrm{h}$ for 6 hours ${ }^{2}$. Admission $\mathrm{SCr}$, defined as the first available value documented 24 hours prior to or 6 hours after ICU admission, was used as baseline as seen in literature ${ }^{8}$. ABW and height were defined as the first available values documented within $24 \mathrm{~h}$ after admission to ICU. IBW was then calculated as previously reported ${ }^{9}$ :

For males: IBW $(\mathrm{kg})=50 \mathrm{~kg}+0.91 *($ Height $[\mathrm{cm}]-152.4)$

For females: IBW $(\mathrm{kg})=45.5 \mathrm{~kg}+0.91 *($ Height $[\mathrm{cm}]-152.4)$ 
For patients whose $A B W \geq 1.3$ times of their IBW, IBW was further adjusted as ${ }^{10}$ :

Adjusted IBW $=\mathrm{IBW}+0.4 *(\mathrm{ABW}-\mathrm{IBW})$

To calculate UO normalized by body weight, we used ABW and IBW separately. Diagnosis of AKI was made when SCr or UO calculated by either ABW or IBW met the aforementioned thresholds ${ }^{11}$. Patients received renal replacement treatment without documented ESRD was also diagnosed AKI. BMI was calculated using $\mathrm{ABW}$ and height and then categorized: underweight $(\mathrm{BMI}<18.5)$, normal $(18.5 \leq \mathrm{BMI}<$ $25)$, overweight $(25 \leq \mathrm{BMI}<30)$, and obese $(\mathrm{BMI} \geq 30)$.

To further illustrate differences among subsets of the patients diagnosed by the two components of KDIGO definition, we divided the patients into following groups: those diagnosed by ABW- but not IBWnormalized $\mathrm{UO}$ or by SCr (referred to as the ABW group), those by IBW- but not ABW-normalized UO or by $\mathrm{SCr}$ (the IBW group), those by SCr alone but not by ABW-/IBW-normalized UO (the SCr group), those by both $\mathrm{ABW}$ - and IBW-normalized UO but not by SCr (the BW group) and those who were not diagnosed with AKI by either ABW-/IBW-normalized UO or SCr (the non-AKI group).

\section{Covariables}

Demographic features such as age, gender and BMI, ethnicity (white, black, Asian, Hispanic and other) were included. Patients' comorbidities, including congestive heart failure, hypertension, diabetes, cancer, obesity, and weight loss as indicated by ICD-9 codes, were extracted and coded as binary variables. Other charted data and laboratory tests within the first day of ICU stay were included as continuous variables: the Sequential Organ Failure Assessment (SOFA) score, white blood cell count and hematocrit, serum sodium, potassium, chloride, bicarbonate, anion gap and glucose and blood urea nitrogen. Admission, highest, lowest and range of creatinine values, whether or not vasopressors, mechanic ventilation or renal replacement treatment were required within the first week of ICU stay were also included.

\section{Outcome Measures}

The primary outcomes were hospital mortality and 90-day mortality. Deaths were recorded in the database and originally identified from hospital records or the Social Security Death Index.

\section{Sensitivity analysis}

To decide the robustness of our results, two sensitivity analyses were carried out. Patients' serum creatinine might have been elevated to an abnormal level and AKI might have occurred before they admitted into ICU, so using the admission creatinine level 24 hours prior to or 6 hours after ICU admission as baseline could be questionable. To address this issue, in our first sensitivity analysis, we only included patients whose admission creatinine levels were within the normal reference ranges (below $1.3 \mathrm{mg} / \mathrm{dl}$ for male and $1.1 \mathrm{mg} / \mathrm{dl}$ for female respectively). In the second sensitivity analysis, sepsis patients were identified based on Angus's proposals ${ }^{12}$, to examine whether the results were any different for this specific subset of patients. 


\section{Statistical analysis}

Continuous variables were reported as medians with interquartile ranges (IQR, 25th-75th percentiles) and categorical variables as counts with percentages. Missing data were imputed using the multiple imputation by chained equations with 5 imputation and 100 iterations. Mann-Whitney $\mathrm{U}$ test and Kruskal-Wallis test were used to compare continuous data, and Fisher's exact test or Pearson's chisquared test to compare categorical data when appropriate. Association between variables were assessed by the Spearman correlation and existence of multicollinearity in models was detected with variance inflation factors (VIFs), correlation coefficients below 0.30 and VIFs below 1.5 were deemed acceptable. Discrepancies between diagnosis of AKI based on different components of KDIGO guidelines were assessed by the McNemar's Chi-squared test and agreement by Cohen's weighted kappa values and agreement percentages. Logistic regression analyses were used to explore and identify independent variables associated with hospital and 90-day mortality. After removing variables that were highly correlated, the left ones were fed into a full model. Then a stepwise removal of non-significant variables from the model until all variables left were significant was carried out base on the Akaike information criterion (AIC). The Bonferroni correction was applied in pairwise comparisons among multiple groups. All statistical analyses were performed using $\mathrm{R}$ software $3.6 .3^{13}$.

\section{Results}

\section{Baseline characteristics}

The MIMIC $\otimes$ database contains 61,532 unique ICU stay records. Of them 10,925 (17.75\%) were removed because patients' age was younger than 18 or older than 89 . To ensure independence between hospital stay records, $14,090(22.90 \%)$ records identified as non-first ICU stays were excluded. 5,586 (9.08\%) patients who stayed less than 24 hours in ICU were also excluded. Diagnosing AKI requires admission serum creatinine, UO and patients' height and weight, so 14,233 (23.13\%) patients lacking this necessary information were discarded. We also excluded 1,973 (3.21\%) patients with previous diagnosis of ESRD. Thus, a total of 14,725 patients was eventually included in the current study (Fig. 1). In all variables included in this study, there was less than $1 \%$ missing data, which was imputed as described in Methods section.

Characteristics and outcomes of the cohort are summarized in Table 1. 8,792 (59.71\%) of the patients were male. Median age was 65 years (IQR 54-76) and median ABW and IBW were $80 \mathrm{~kg}$ (IQR 68-94) and $70 \mathrm{~kg}$ (IQR 61-78) respectively. 10,601 (72.00\%) patients were white and 11,189 (75.99\%) came to ICU as emergency admission. Median of first-day SOFA score was 2 (IQR 4-6). 7,362 (50.00\%) patients received vasopressors treatment during their ICU stay. Mechanical ventilation was used in 9,432 (64.05\%) of the patients. Hospital mortality was $8.82 \%$ among the cohort, while 90 -day mortality was $14.50 \%$.

\section{AKI diagnosis and staging}


AKI was found in 4,298 (29.19\%) and 3,060 (20.78\%) patients when ABW or IBW was used to calculate hourly UO, respectively. Apart from that, AKI occurred in 3,551 (24.12\%) patients according to their SCr change. Taken together, a total of 6,033 (40.97\%) patients were diagnosed with AKI when SCr and ABWnormalized urine volume were considered and 5,152 (34.99\%) patients when considering SCr and IBWnormalized urine volume. In both scenarios, more patients were identified by ABW than by IBW, which were confirmed by McNemar's Chi-squared tests $(p<0.001)$. In addition, AKI stages also differed between these patients $(p<0.001)$ with a kappa of $0.87(95 \% \mathrm{Cl}, 0.86 \sim 0.88)$ and an agreement percentage of $90.40 \%$ (Table 2).

Among those diagnosed with AKI according to either SCr or hourly UO, 1,443 patients were diagnosed by $\mathrm{SCr}, \mathrm{ABW}$ - and IBW-normalized UO at the same time, while 1,535 were diagnosed by both ABW-/IBWnormalized UO but not $\mathrm{SCr}$ (the BW group), 947 by ABW only but not IBW or SCr (the ABW group), 66 by IBW only but not ABW or SCr (the IBW group) and 1,719 by SCr but not ABW-/IBW-normalized UO (the SCr group). 8,626(58.58\%) patients with no evidence of AKI were defined as the non-AKI group (Fig. 2).

\section{Association between AKI defined by different body weights and mortality}

As the incidence of $\mathrm{AKI}$ in $\mathrm{ABW}$ group was higher than that in other groups, we assumed that using $\mathrm{ABW}$ instead of IBW tended to underestimate patients' hourly UO, resulting in these patients more likely to be identified as AKI. Thus, details about the AKI defined by different body weight and mortality were summarized in Additional file 1 Table S1.

The hospital and 90-day mortality of the ABW group were significantly lower than that of the BW group and the $\mathrm{SCr}$ group $(\mathrm{p}<0.001)$. Interestingly, no differences were found in hospital and 90 -day mortality between ABW group and non-AKI group. This indicated that a subset of patients was overestimated as AKI due to lower UO calculated from heavier body weights, while their hospital and 90-day risks of death were in fact not different from non-AKI patients. We also found significantly higher hospital and 90-day mortality in BW and SCr group compared with either ABW or non-AKI group (all $p<0.001$ ), while no differences were found between the two groups, suggesting that the subset of patients identified by SCr or both $A B W$ and IBW were at greater risks of hospital and 90-day death than non-AKI patients or those identified by $A B W$ alone. To our surprise, SOFA scores of patients identified as AKI by ABW were significantly higher compared with non-AKI patients $(p<0.001)$ but not significantly different from patients diagnosed with AKI by both ABW and IBW $(p=0.19)$.

Taken together, using ABW to calculate $\mathrm{UO}$ tended to overestimate AKI incidence in patients with heavier body weight. While in fact risks of hospital or 90-day death of these patients were significantly lower than those diagnosed by SCr or UO calculated from both ABW and IBW and not significantly different from non-AKI critical ill patients.

\section{Logistic regression}


Univariable and multivariable logistic regression models were then constructed to decide independent risks factors. Using non-AKI group as reference, our first model containing only the group variable (hereafter referred to as the raw model), revealed that BW group and $\mathrm{SCr}$ group were significantly related to higher hospital and 90-day mortality while IBW group was found to be associated with 90-day but not hospital mortality (Table 3 ). We also noticed that ABW group was associated with neither hospital mortality nor 90-day mortality, while both $\mathrm{BW}$ and $\mathrm{SCr}$ group had odds ratios of over 2 in regards to hospital mortality compared with the non-AKI group. In the second model, age, gender and BMI were included besides the group variable (the adjusted model). In this model, similar to the raw model, while both BW group and SCr group were associated with significantly higher hospital and 90-day mortality, ABW and IBW group were not. The highest, the lowest, admission, change of creatinine and the first BUN levels within the first 7 days in ICU were all found to be highly correlated, so did the time it took to reach the highest and lowest creatinine, blood sodium and chloride levels, blood chloride and bicarbonate levels, as well as bicarbonate and anion gap levels. Thus, variables including the highest, the first, the lowest and the change of creatinine levels, the time it took to reach the lowest creatinine, blood BUN, chloride and anion gap levels were dropped and then a model including all the remaining variables were constructed (the full model). Though odds ratios shrank slightly after being adjusted by more variables, still we found that BW group and SCr group were linked to significantly higher mortalities compared with non-AKI group while neither ABW group nor IBW group was. Finally, a stepwise model excluding variables that were not significant in the full model was built (the reduced model). The reduced model showed similar results to above models, where only BW group and $\mathrm{SCr}$ group but not ABW group and IBW group were found to be linked to higher risks of death compared with non-AKI group.

\section{Sensitivity analysis}

When limiting patients to those with admission creatinine within the normal range, 4,300 patients with abnormal creatinine levels at ICU admission (above $1.3 \mathrm{mg} / \mathrm{dL}$ for male or $1.1 \mathrm{mg} / \mathrm{dL}$ for female) were dropped and a cohort containing the left 10,425 patients was analyzed. Four logistic regression models were built following same procedures as mentioned earlier. The results still demonstrated that both BW and $\mathrm{SCr}$ groups were linked to higher hospital and 90-day mortality while ABW and IBW group were not (see Additional file 2 Table S1).

When the patient cohort was limited to those with ICD-9 codes indicating sepsis during their ICU stay as proposed by Angus et al ${ }^{12}$, a total of 3,365 cases were identified. Similar results were observed: significant higher risks of hospital and 90-day mortality were found in BW group and $\mathrm{SCr}$ group but not in ABW or IBW group (see Additional file 2 Table S2).

Consistently, logistic regression analyses demonstrated that ABW group was not linked to a different mortality compared with non-AKI group. In contrary, patients in BW group and SCr group were at significantly higher risks of hospital and 90-day death compared with those of the non-AKI group. Results were further confirmed in two separate sensitivity analyses where sepsis patients or patients with normal admission creatinine levels were considered. 


\section{Discussion}

In the current study, we examined the differences in hospital and 90-day mortality of critical ill patients diagnosed as AKI by SCr change or UO normalized by different definitions of body weight. ABWnormalized UO identified over 1,000 AKI patients than IBW did, accounting for more than $8 \%$ of the whole cohort in this study. However, results showed that these patients had similar risks of hospital and 90-day mortality to that of patients with no evidence of AKI. On the contrary, AKI patients identified by SCr or by both $A B W$ and IBW had over twice higher the risks of hospital death, 1.7 and 1.4 times higher the risks of 90-day death respectively compared with non-AKI patients. Our results revealed that using ABW led to underestimate of patients' UO, subsequently overestimation of AKI.

There have been studies focusing on diagnosis of AKI by different definitions of body weight. Thongprayoon et a/ reported similar results in a single center, retrospective study of 493 ICU patients ${ }^{4}$. In their study, AKI patients identified by both ABW and IBW had significant higher 90-day mortality (OR 1.76, 95\% Cl 1.05-2.95) compared with patients who didn't had AKI, while patients who had AKI according to ABW but not IBW had no significant increase in the risk of 90-day mortality. They concluded that ABW provided better sensitivity and earlier recognition of $A K I$ and thus proposed that $U O$ normalized by ABW should be used in clinical practice for screening purposes, while UO normalized by IBW should be used in researches focusing on interventions for AKI patients. In another study by Katayama et a/ where 569 septic patients were studied, the authors found a discrepancy rate of $7.6 \%$ in terms of the urinary diagnosis of AKI diagnosed by ABW and IBW, while no difference in 90-day mortality was found between the two groups of patients ${ }^{5}$. However, in Thongprayoon's study, although baseline characteristics displayed in their paper included several basic demographics and comorbidities, the researchers failed to provide details about whether there were any differences in these factors, of which some might well be potential confounders, between subsets of patients. As a matter of fact, odds ratios the researchers reported were only adjusted by age and APACHE score, whereas BMI, which had apparently influence on odds ratios as mentioned in their paper, was not included. In addition, to assess the sensitivity and specificity of ABW and IBW in diagnosing AKI, SCr was used as reference standard. Although $\mathrm{SCr}$ has been widely used to estimate glomerular filtration rate (GFR), it is believed to be affected by various factors including medication use, fluid balance et al ${ }^{14,15}$. Whether body mass could have affected $\mathrm{SCr}$ was not excluded as aforementioned in their study. In addition, fluid overload is common in ICU, which also challenged the notion to use $\mathrm{SCr}$ as the reference standard to evaluate the performance of $\mathrm{ABW}$ and IBW in Thongprayoon's study ${ }^{14,16,17}$. It's necessary to point out that in Katayama's study, they only included subjects identified as sepsis by the Third International Consensus Definitions for Sepsis and Septic Shock (Sepsis-3), which explained why 90-day mortality were much higher than that in Thongprayoon's and in our study. This might be the reason why no difference was found between patients diagnosed AKI by ABW and IBW their study.

Using data from the MIMIC $₫$ database, we found in a large cohort of over 14,000 patients that normalizing UO using ABW led to significantly more AKI cases. In contrary to previous studies where it was believed to be accompanied by higher sensitivity, no differences in hospital or 90-day mortality were 
found between patients identified as AKI by ABW only and patients without evidence of AKI, indicating overdiagnosis of AKI due to their heavier body weights. However, SOFA scores of patients identified as AKI by ABW were significantly higher than those of non-AKI patients and similar to those of the patients identified as AKI by both ABW and IBW. The discrepancy between SOFA score and mortality seemed to support the obesity paradox phenomenon where mortality tends to drop as body weight increases, which however, is still controversial ${ }^{18-21}$. While numerous researches indicated that obesity was associated with a higher risk of developing AKI in various patient populations, there was no clear answer as to whether and how obesity contributed to short- and long-term survival of the critical ill ${ }^{8,22-30}$. Although beyond the scope of the current study to elucidate the mechanism underlying the relationship with obesity and mortality, our finding seems to be in accordance with another study where survival benefit due to obesity was evident among patients with more disease severity ${ }^{31}$. While traditionally attributed to high metabolic reserve, this survival benefit was also suggested to be partially by mediated oxidative stress $^{32}$. On the other hand, more researchers thought poor study design, heterogeneous patient populations and statistical bias due to the observational nature of most studies focusing on this topic were to blame ${ }^{21,33}$. Though the reason why outcomes of patients with heavier body weights and higher SOFA scores were similar to those without evidence of AKI remained to be studied, our results clearly demonstrated that using $A B W$ led to underestimation of $U O$ and thus overestimation of AKI. Considering the intensive monitoring and care AKI patients need, any overdiagnosis of AKI could result in unnecessary intervention which might expose patients to potential injury.

There are several limitations in our study. First, the retrospective nature of the current study should be addressed. Although we've tried our best to include all potential clinically relevant factors, other confounding factor not captured could not be ruled out. As the data came from a single-center academic tertiary medical center, generalizability of our results also remains to be further studied. And although our findings indicated that using ABW to diagnose AKI led to overestimate of AKI and patients identified as AKI by ABW alone had similar hospital and 90-day mortality to those without evidence of AKI, causal relationship could not be established since data was not derived from a randomized controlled trial (RCT). However, our study included over 14,000 patients over a period of ten years and results were confirmed in two sensitivity analysis. Given such a large sample size and findings held true in subsequent sensitivity analysis, our results are not likely to alter in future studies. Second, due to relatively small group size of the IBW group ( $\mathrm{N}=66)$, all statistical comparisons between IBW and another group tended to yield non-statistically significant difference. However, in order to examine the difference between ABW and IBW-based AKI diagnosis, the best practice would be to separately consider patients identified as AKI by two different definitions of body weight. As a result, the use of IBW alone to calculate UO for AKI diagnosis was not fully studied in the current study and requires further investigation.

\section{Conclusion}

In this study, we found that using ABW to calculate $U O$ for diagnosis of AKI resulted in underestimation of $\mathrm{UO}$ and overestimation of AKI, especially for obese patients. To avoid overdiagnosis and subsequently 

be accurate.

\section{Declarations}

\section{Ethics approval and consent to participate}

Not applicable.

\section{Consent for publication}

Not applicable.

\section{Availability of data and materials}

All data used in this study was retrived from the MIMIC- $₫$ database, which is freely accessible at https://mimic.physionet.org/. Accessed 23 Nov 2020.

\section{Competing interests}

The authors declare that they have no conflict of interest.

\section{Funding}

Not applicable.

\section{Authors' contributions}

Jiang Jun and Zhang Jing conceived the original idea. Jiang Jun extract all data used in the study and performed statistical analysis. Liu Ye and Xu Dongxue designed data analysis strategy and performed data pre-processing. The main text was first drafted by Jiang Jun and discussed with Zhang Jing, Liu Ye and Xu Dongxue. Peng Zhiyong led and project, reviewed and revised the manuscript. All authors read and approved the final manuscript.

\section{Acknowledgements}

Not applicable. 


\section{References}

1 Hoste, E. A. et al. Epidemiology of acute kidney injury in critically ill patients: the multinational AKI-EPI study. Intensive Care Med41, 1411-1423, doi:10.1007/s00134-015-3934-7 (2015).

2 Kellum, J. A. et al. Kidney disease: Improving global outcomes (KDIGO) acute kidney injury work group. KDIGO clinical practice guideline for acute kidney injury. Kidney International Supplements2, 1-138, doi:10.1038/kisup.2012.1 (2012).

3 Jörres, A. et al. A European Renal Best Practice (ERBP) position statement on the Kidney Disease Improving Global Outcomes (KDIGO) Clinical Practice Guidelines on Acute Kidney Injury: part 2: renal replacement therapy. Nephrology Dialysis Transplantation28, 2940-2945, doi:10.1093/ndt/gft297 (2013).

4 Thongprayoon, C., Cheungpasitporn, W., Akhoundi, A., Ahmed, A. H. \& Kashani, K. B. Actual versus ideal body weight for acute kidney injury diagnosis and classification in critically III patients. BMC Nephrology15, 176, doi:10.1186/1471-2369-15-176 (2014).

5 Katayama, S. et al. Body weight definitions for evaluating a urinary diagnosis of acute kidney injury in patients with sepsis. BMC Nephrology19, 101, doi:10.1186/s12882-018-0895-4 (2018).

6 Ratanapo, S. Effects of type of body weight type on acute kidney injury diagnosis. Saudi Journal of Kidney Diseases and Transplantation27, 627-627, doi:10.4103/1319-2442.182449 (2016).

7 Johnson, A. E. W. et al. MIMIC-III, a freely accessible critical care database. Scientific Data3, 160035, doi:10.1038/sdata.2016.35 (2016).

8 Danziger, J. et al. Obesity, Acute Kidney Injury, and Mortality in Critical Illness. Critical Care Medicine44, 328-334, doi:10.1097/CCM.0000000000001398 (2016).

9 McCarron, M. M. \& Devine, B. J. Clinical Pharmacy: Case Studies: Case Number 25 Gentamicin Therapy. Drug Intelligence \& Clinical Pharmacy8, 650-655, doi:10.1177/106002807400801104 (1974).

10 Erstad, B. L. Dosing of medications in morbidly obese patients in the intensive care unit setting. Intensive Care Medicine30, 18-32, doi:10.1007/s00134-003-2059-6 (2004).

11 Johnson, A. E. W., Stone, D. J., Celi, L. A. \& Pollard, T. J. The MIMIC Code Repository: enabling reproducibility in critical care research. Journal of the American Medical Informatics Association25, 3239, doi:10.1093/jamia/ocx084 (2017).

12 Angus, D. C. et al. Epidemiology of severe sepsis in the United States: Analysis of incidence, outcome, and associated costs of care. Critical Care Medicine29 (2001).

13 R: A Language and Environment for Statistical Computing (R Foundation for Statistical Computing, R Foundation for Statistical Computing, Vienna, Austria., 2019). 
$14 \mathrm{Liu}, \mathrm{K}$. D. et al. Acute kidney injury in patients with acute lung injury: Impact of fluid accumulation on classification of acute kidney injury and associated outcomes*:. Critical Care Medicine39, 2665-2671, doi:10.1097/CCM.0b013e318228234b (2011).

15 Moledina, D. G. \& Parikh, C. R. Phenotyping of Acute Kidney Injury: Beyond Serum Creatinine. Seminars in Nephrology38, 3-11, doi:https://doi.org/10.1016/j.semnephrol.2017.09.002 (2018).

16 Yacoub, H. et al. Acute kidney injury adjusted to volume status in critically ill patients: recognition of delayed diagnosis, restaging, and associated outcomes. Int J Nephrol Renovasc Dis9, 257-262, doi:10.2147/ijnrd.S113389 (2016).

17 Macedo, E. et al. Fluid accumulation, recognition and staging of acute kidney injury in critically-ill patients. Critical Care14, R82, doi:10.1186/cc9004 (2010).

18 Christopher, K. B. The Body Mass Index Paradox*. Critical Care Medicine43 (2015).

19 Kramer, A. A. A Different Type of "Obesity Paradox"*. Critical Care Medicine47 (2019).

20 Deliberato, R. O. et al. An Evaluation of the Influence of Body Mass Index on Severity Scoring*. Critical Care Medicine47 (2019).

21 Schiffl, H. \& Lang, S. M. Obesity, acute kidney injury and outcome of critical illness. International Urology and Nephrology49, 461-466, doi:10.1007/s11255-016-1451-4 (2017).

22 Lu, J. L., Kalantar-Zadeh, K., Ma, J. Z., Quarles, L. D. \& Kovesdy, C. P. Association of Body Mass Index with Outcomes in Patients with CKD. Journal of the American Society of Nephrology25, 2088-2096, doi:10.1681/ASN.2013070754 (2014).

23 Sakr, Y. et al. Being Overweight Is Associated With Greater Survival in ICU Patients: Results From the Intensive Care Over Nations Audit. Critical Care Medicine43, 10 (2015).

24 Grigorian, A. et al. Black Race and Body Mass Index Are Risk Factors for Rhabdomyolysis and Acute Kidney Injury in Trauma. Journal of Investigative Surgery, 1-8, doi:10.1080/08941939.2018.1493162 (2018).

25 Ju, S. et al. Body Mass Index as a Predictor of Acute Kidney Injury in Critically III Patients: A Retrospective Single-Center Study. Tuberc Respir Dis81, 311, doi:10.4046/trd.2017.0081 (2018).

26 Wong, E. S. et al. Examining the BMI-mortality relationship using fractional polynomials. BMC Medical Research Methodology11, 175, doi:10.1186/1471-2288-11-175 (2011).

27 Wang, H. et al. Higher body mass index is not a protective risk factor for 28-days mortality in critically ill patients with acute kidney injury undergoing continuous renal replacement therapy. Renal Failure41, 726-732, doi:10.1080/0886022X.2019.1650767 (2019). 
28 Pedersen, A. B., Gammelager, H., Kahlert, J., Sørensen, H. T. \& Christiansen, C. F. Impact of body mass index on risk of acute kidney injury and mortality in elderly patients undergoing hip fracture surgery. Osteoporosis Internationa/28, 1087-1097, doi:10.1007/s00198-016-3836-8 (2017).

29 Druml, W., Metnitz, B., Schaden, E., Bauer, P. \& Metnitz, P. G. Impact of body mass on incidence and prognosis of acute kidney injury requiring renal replacement therapy. Intensive Care Med36, 1221-1228, doi:10.1007/s00134-010-1844-2 (2010).

30 Chao, C. T. et al. Impact of body mass on outcomes of geriatric postoperative acute kidney injury patients. SHOCK41, 400-405, doi:10.1097/shk.0000000000000143 (2014).

$31 \mathrm{Kim}, \mathrm{H}$. et al. The impact of disease severity on paradoxical association between body mass index and mortality in patients with acute kidney injury undergoing continuous renal replacement therapy. $B M C$ Nephrology19, 32, doi:10.1186/s12882-018-0833-5 (2018).

32 Billings, F. T. et al. Obesity and Oxidative Stress Predict AKI after Cardiac Surgery. Journal of the American Society of Nephrology23, 1221-1228, doi:10.1681/ASN.2011090940 (2012).

33 Liu, Y. Y., Xue, F. S. \& Li, H. X. Assessing impact of body mass index on risk of acute kidney injury and mortality in elderly patients undergoing hip fracture surgery. Osteoporosis Internationa/28, 3515-3516, doi:10.1007/s00198-017-4194-x (2017).

\section{Tables}

Due to technical limitations, table 1 to 3 is only available as a download in the Supplemental Files section.

\section{Figures}




\section{MIMIC III data base $(n=61,532)$}

Age $<18$ or $>89(n=10,925)$

Not first ICU stay $(n=14,090)$

ICU stay shorter than 24 hours $(n=5,586)$

Height missing $(n=10,687)$

Body weight missing $(n=473)$

No chart data available $(n=246)$

No admission creatinine $(n=439)$

Creatinine or urine output records less

than $2(n=1,535)$

\section{End stage renal disease $(n=2,006)$}

\section{Study cohort $(n=14,725)$}

\section{Figure 1}

Flow chart of patient selection process. The MIMIC- $₫$ v 1.4 includes a total of 61532 ICU admission records. A final cohort of 14725 were identified eligible and included in this study. MIMIC, Medical Information Mart for Intensive Care; ICU, intensive care unit. 


\section{MIMIC III data base $(n=61,532)$}

Age $<18$ or $>89(n=10,925)$

Not first ICU stay $(n=14,090)$

ICU stay shorter than 24 hours $(n=5,586)$

Height missing $(n=10,687)$

Body weight missing $(n=473)$

No chart data available $(n=246)$

No admission creatinine $(n=439)$

Creatinine or urine output records less

than $2(n=1,535)$

\section{End stage renal disease $(n=2,006)$}

\section{Study cohort $(n=14,725)$}

\section{Figure 1}

Flow chart of patient selection process. The MIMIC- $₫$ v 1.4 includes a total of 61532 ICU admission records. A final cohort of 14725 were identified eligible and included in this study. MIMIC, Medical Information Mart for Intensive Care; ICU, intensive care unit. 
MIMIC III data base $(n=61,532)$

Age $<18$ or $>89(n=10,925)$

Not first ICU stay $(n=14,090)$

ICU stay shorter than 24 hours $(n=5,586)$

Height missing $(n=10,687)$

Body weight missing $(n=473)$

No chart data available $(n=246)$

No admission creatinine $(n=439)$

Creatinine or urine output records less

than $2(n=1,535)$

End stage renal disease $(n=2,006)$

Study cohort $(n=14,725)$

Figure 1

Flow chart of patient selection process. The MIMIC- $₫$ v 1.4 includes a total of 61532 ICU admission records. A final cohort of 14725 were identified eligible and included in this study. MIMIC, Medical Information Mart for Intensive Care; ICU, intensive care unit. 


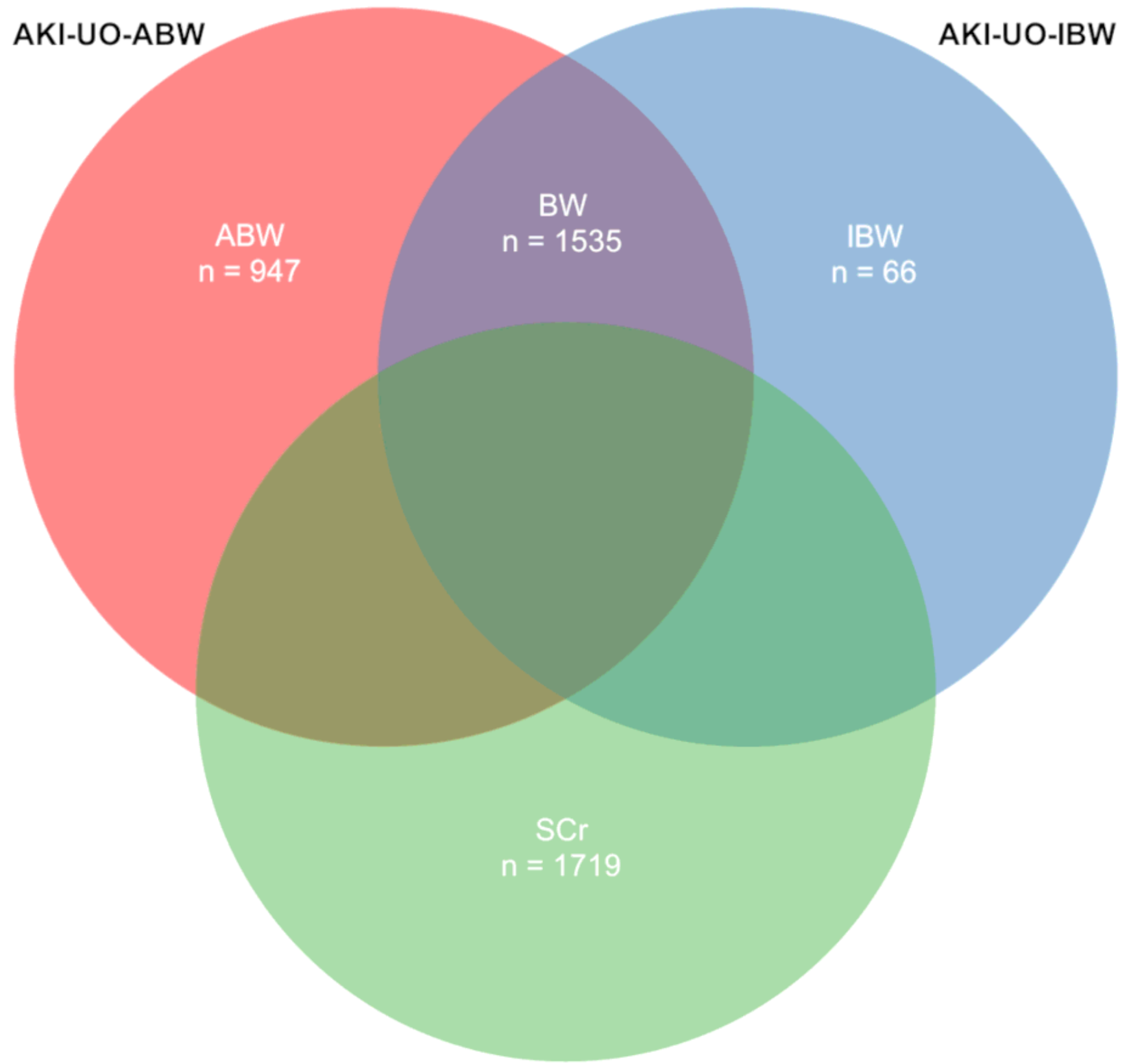

$\mathrm{AKI}-\mathrm{SCr}$

\section{Figure 2}

AKI patients identified by different components of the KDIGO guidelines and grouping of patient. 947 patients were diagnosed as AKI by ABW only but not IBW or SCr (AKI-UO-ABW, the ABW group), 66 by IBW only but not ABW or SCr (AKI-UO-BW, the IBW group) and 1,719 by SCr but not ABW-/IBW-normalized UO (AKI-SCr, the SCr group). AKI, acute kidney injury; ABW, actual body weight; IBW, ideal body weight; UO, urine output; BW, body weight; SCr, serum creatinine. 


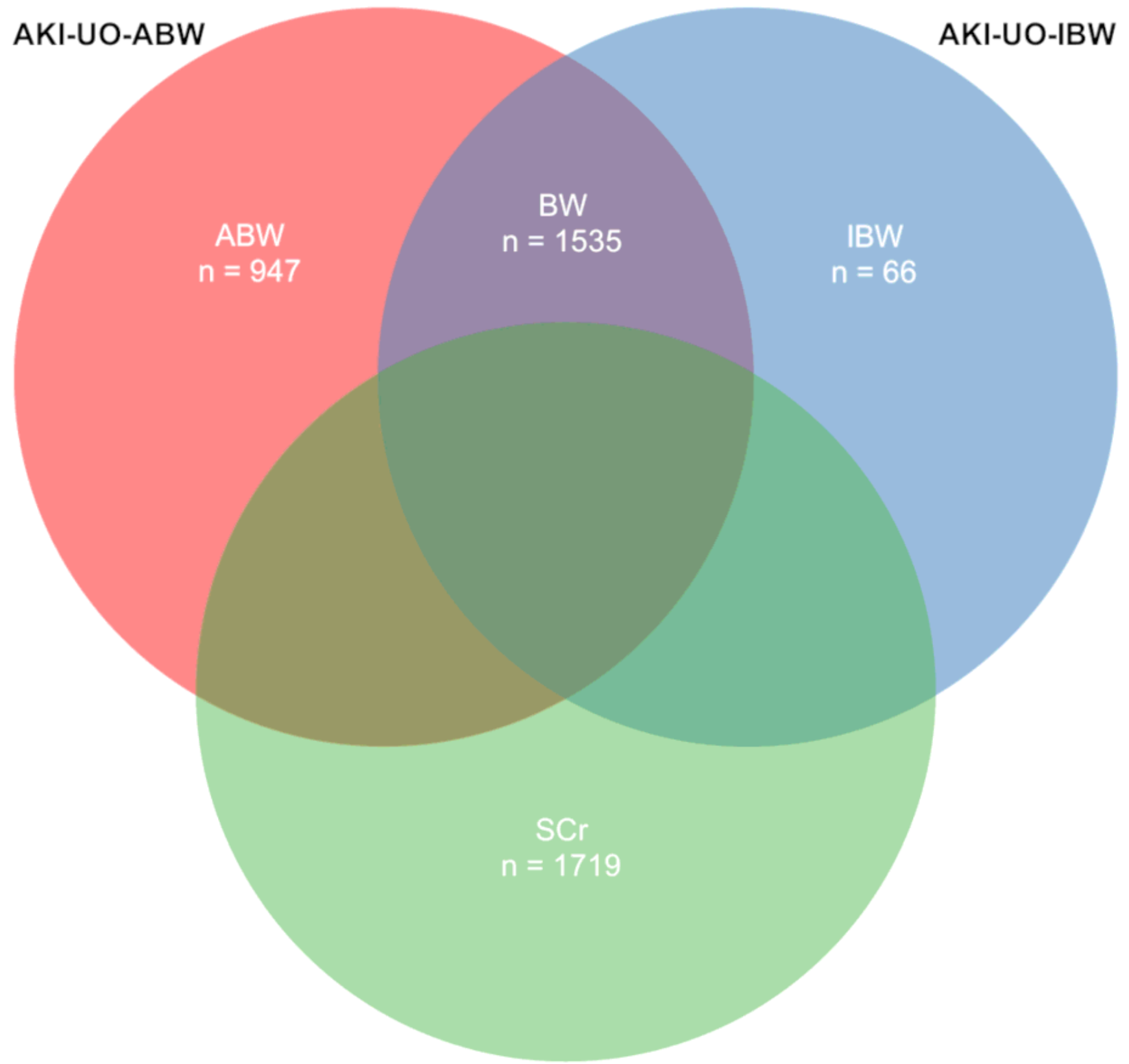

$\mathrm{AKI}-\mathrm{SCr}$

\section{Figure 2}

AKI patients identified by different components of the KDIGO guidelines and grouping of patient. 947 patients were diagnosed as AKI by ABW only but not IBW or SCr (AKI-UO-ABW, the ABW group), 66 by IBW only but not ABW or SCr (AKI-UO-BW, the IBW group) and 1,719 by SCr but not ABW-/IBW-normalized UO (AKI-SCr, the SCr group). AKI, acute kidney injury; ABW, actual body weight; IBW, ideal body weight; UO, urine output; BW, body weight; SCr, serum creatinine. 


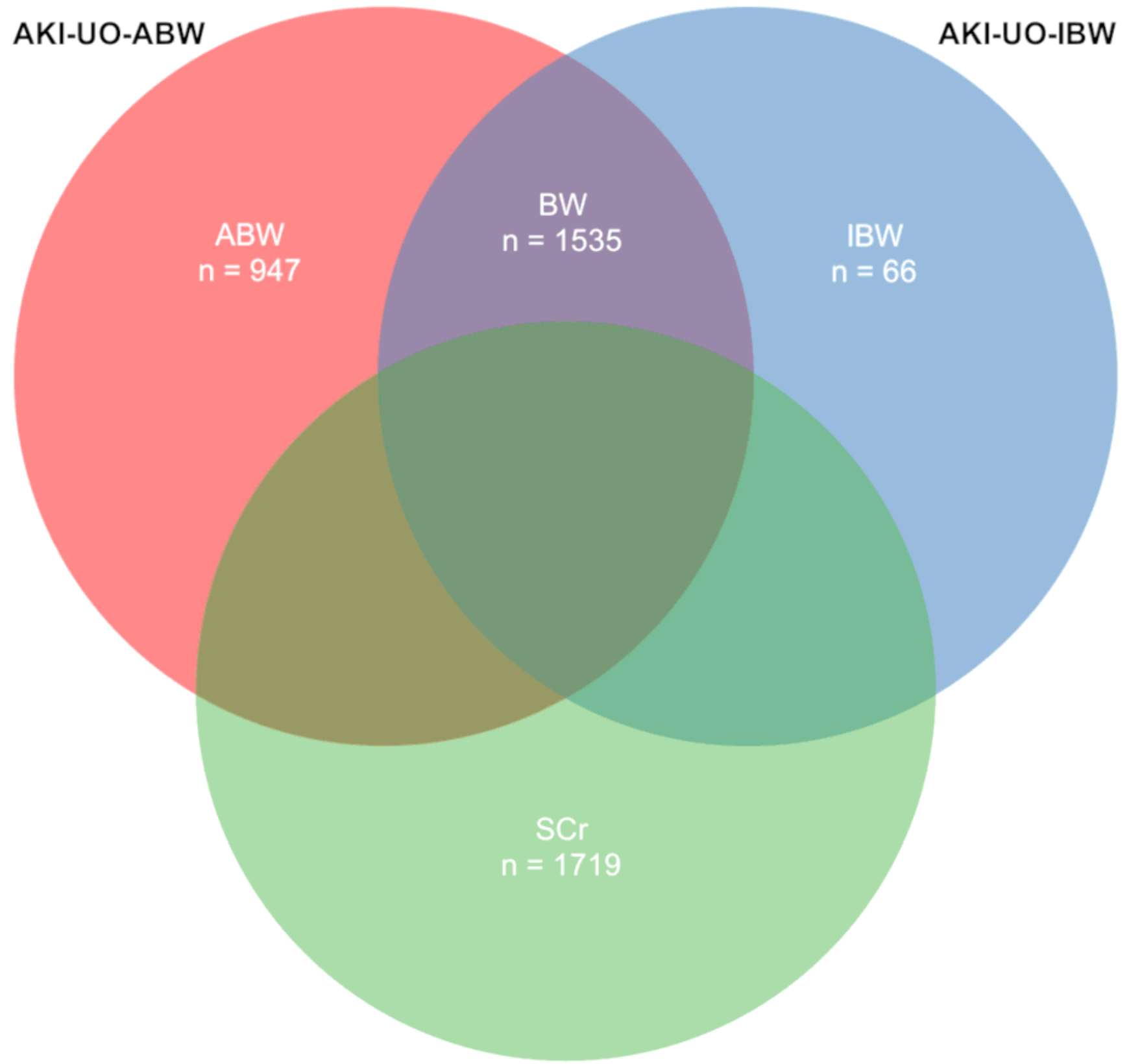

$\mathrm{AKI}-\mathrm{SCr}$

\section{Figure 2}

AKI patients identified by different components of the KDIGO guidelines and grouping of patient. 947 patients were diagnosed as AKI by ABW only but not IBW or SCr (AKI-UO-ABW, the ABW group), 66 by IBW only but not ABW or SCr (AKI-UO-BW, the IBW group) and 1,719 by SCr but not ABW-/IBW-normalized UO (AKI-SCr, the SCr group). AKI, acute kidney injury; ABW, actual body weight; IBW, ideal body weight; UO, urine output; BW, body weight; SCr, serum creatinine. 


\section{Supplementary Files}

This is a list of supplementary files associated with this preprint. Click to download.

- AddtionalFiles1.xls

- AddtionalFiles1.xls

- AddtionalFiles1.xls

- AddtionalFiles2.xls

- AddtionalFiles2.xls

- AddtionalFiles2.xls

- Table1.Patient.Characteristics.xlsx

- Table1.Patient.Characteristics.xIsx

- Table1.Patient.Characteristics.xlsx

- Table2.AKI.Stages.xls

- Table2.AKI.Stages.xls

- Table2.AKI.Stages.xls

- Table3.LogisticRegressionResults.xls

- Table3.LogisticRegressionResults.xls

- Table3.LogisticRegressionResults.xls 